\title{
Motion of Stellar Particles and Photons within Spherical Liquid Stars
}

\author{
Chifu E. Ndikilar ${ }^{1}$, Amusa S. Kolawole ${ }^{2}$ and Hafeez Y. Hafeez ${ }^{3}$ \\ 'Department of Physics, Federal University Dutse, P.M.B 7156, Dutse, Jigawa State, Nigeria; \\ ebenechifu@yahoo.com \\ 2Department of Physics, Gombe State University, Gombe, Nigeria; \\ sheriff.amusa77@gmail.com \\ ${ }^{3}$ SRM Research Institute, SRM University, Kattankulathur, Chennai - 603203, Tamil Nadu, India; \\ hafeezyusufhafeez@gmail.com
}

\begin{abstract}
Objectives: The motion of test particles and photons within a spherical liquid star is studied. Equations of motion of test particles in the star's interior and equatorial plane are derived. The general relativity interior metric tensor for a spherical liquid star and tensor analysis is used to study the motion of particles and photons within the star. Methods: The obtained geodesic equations of motion for stellar particles are explicitly space coordinate and coordinate proper time derivatives. This differs from Schwarz child's equations of motion in a gaseous field. Findings: Along the equatorial plane of the liquid star, the equations of motion have additional terms not found in Schwarz child's field and in Newtonian theory. The equation of motion of a photon in this gravitational field differs from that of Schwarz child's mass point field by the definition of its Hilbert radius. Applications: This article thus initiates the study of the motion of stellar particles and photons within spherical liquid stars in the framework of the General Theory of Relativity which hitherto has not been exploited.
\end{abstract}

Keywords: Geodesic, Motion, Photons, Spherical Liquid Stars, Stellar Particles

\section{Introduction}

In the conventional approach to the study of stars in the universe, the stars are considered to be gaseous spheres in nature; consisting mainly of hydrogen and a very inhomogeneous interior ${ }^{1}$. A liquid star model was proposed in the 1920 ' $\mathrm{s}^{2}$ in which the internal constitution of stars is much populated. Though, there were many astrophysical evidences of liquid stars, this model was not convincing to the scientific community as it lacked a solid mathematical basis. This theory was based on observational analysis and arguments rather than a mathematical model.
An absolutely different approach to the problem was introduced in 2014. This approach is based on the simultaneous consideration of a liquid star and its field according to the General Theory of Relativity (which is the most accepted modern theory of gravitation introduced by Einstein in 1916). Liquid stars are considered as this matches certain new observational evidences for the state of condensed matter inside stars; in particular that the Sun consists of high-temperature liquid metallic hydrogen ${ }^{4}$. In this article, the motion of stellar particles and photons within spherical liquid stars is studied in the framework of the General Theory of Relativity ${ }^{3,5}$.

${ }^{*}$ Author for correspondence 


\section{Metric Tensor of a Spherical Liquid Star}

Fields of spherical liquid stars can be considered to be spherically symmetric such that the structure, matter, and field of such a star can be defined in terms of a spherically symmetric space (space-time) metric. Among the spacetime metrics of General Relativity Theory, three primary metrics describe spherically symmetric gravitational fields. These are Schwarzschild metric of a mass-point $\underline{\underline{6}}$, Schwarzschild metric of a sphere filled with incompressible liquid ${ }^{7}$ and de Sitter's metric which describes a spherical distribution of physical vacuum $\stackrel{8}{\text {. }}$

Schwarzschild mass-point metric describes the field of a spherically symmetric massive body to so large a distance from it that the physical size of the body is neglected and space does not rotate or deform ${ }^{6}$.

De Sitter's metric on the other hand, describes a space filled with a spherically symmetric homogeneous distribution of physical vacuum without any island of mass. The physical vacuum has a very low density and the metric manifest in such a way that the space does not deform and rotate ${ }^{8}$.

The metric of a space filled with an incompressible fluid was introduced by Schwarzschild ${ }^{7}$ in a truncated form containing a lot of limitations. He re-imposed the limitations in the process of deriving the metric in order to avoid breaking in the field. The most complete form of the metric that is free of limitations and takes space breaking into consideration was introduced in 2009 3.5 . The metric of a space filled with an incompressible fluid in its complete form in spherical polar coordinates is given as:

$d s^{2}=\frac{1}{4}\left(3 \sqrt{1-\frac{r_{g}}{a}}-\sqrt{1-\frac{r^{2} r_{g}}{a^{3}}}\right)^{2} c^{2} d t^{2}-\frac{d r^{2}}{1-\frac{r^{2} r_{g}}{a^{3}}}-r^{2}\left(d \theta^{2}+\sin ^{2} \theta d \phi^{2}\right)$

where $a$ is the physical radius of the sphere, $r_{g}=2 G M / c^{2}$ is the Hilbert radius of the massive body and $M$ is the spherical body's mass (which is the mass of the field source). This metric is the most suitable for studying spherical liquid stars in General Relativity Theory.

If $r<a$, the metric is the internal metric of the sphere filled with an incompressible fluid. At the surface $r=a$ the metric coincides with that of a mass point. Also, the outer metric is the same as that of the mass point.

From equation (1), we can deduce the contra variant metric tensor using the quotient theorem of tensor analysis and formulate the non-vanishing coefficients of affine connection in their complete form ${ }^{9}$ as:

$$
\begin{aligned}
& \Gamma_{11}^{1}=\frac{r r_{g}}{a^{3}}\left(1-\frac{r^{2} r_{g}}{a^{3}}\right)^{-1} \\
& \Gamma_{22}^{1}=-r\left(1-\frac{r^{2} r_{g}}{a^{3}}\right) \\
& \Gamma_{33}^{1}=r\left(1-\frac{r^{2} r_{g}}{a^{3}}\right) \sin ^{2} \theta
\end{aligned}
$$

$$
\Gamma_{12}^{2}=\frac{1}{r}
$$

$$
\Gamma_{33}^{2}=-\sin \theta \cos \theta
$$

$$
\Gamma_{23}^{3}=\cot \theta
$$

$$
\Gamma_{13}^{3}=\frac{1}{r}
$$

$$
\Gamma_{01}^{0}=\frac{\frac{r r_{g}}{a^{3}}\left(1-\frac{r^{2} r_{g}}{a^{3}}\right)^{-1 / 2}}{3\left(1-\frac{r_{g}}{a}\right)^{1 / 2}-\left(1-\frac{r^{2} r_{g}}{a^{3}}\right)^{1 / 2}}
$$




\section{Motion of Stellar Particles within a Spherical Liquid Star}

General Relativistic equation of motion for particles of non-zero rest mass in a gravitational field ${ }^{10,11}$ is given as:

$$
\frac{d^{2} x^{\mu}}{d \tau^{2}}+\Gamma_{v \lambda}^{\mu}\left(\frac{d x^{v}}{d \tau}\right)\left(\frac{d x^{\lambda}}{d \tau}\right)=0
$$

Where $\tau$ is a proper time.

The equations of motion are therefore obtained explicitly as follows:

Firstly, setting $\mu=0$ in Equation (3) yields:

$$
\frac{d^{2} x^{0}}{d \tau^{2}}+\Gamma_{v \lambda}^{0}\left(\frac{d x^{v}}{d \tau}\right)\left(\frac{d x^{\lambda}}{d \tau}\right)=0
$$

or

$$
\frac{d^{2} x^{0}}{d \tau^{2}}+\Gamma_{01}^{0}\left(\frac{d x^{0}}{d \tau}\right)\left(\frac{d x^{1}}{d \tau}\right)=0
$$

or

$$
\frac{d^{2}}{d \tau^{2}}(c t)+\Gamma_{01}^{0}\left(\frac{d r}{d \tau}\right)\left(\frac{d}{d \tau} c t\right)=0
$$

or

$$
c \ddot{t}+\Gamma_{01}^{0} \dot{r} \dot{c} \dot{t}=0
$$

Substituting Equation (2h) into Equation (7) gives:

$$
c \ddot{t}+\frac{c \dot{r} t \frac{r r_{g}}{a^{3}}\left(1-\frac{r^{2} r_{g}}{a^{3}}\right)^{-1 / 2}}{3\left(1-\frac{r_{g}}{a}\right)^{1 / 2}-\left(1-\frac{r^{2} r_{g}}{a^{3}}\right)^{1 / 2}}=0
$$

or

$$
\left[3\left(1-\frac{r_{g}}{a}\right)^{1 / 2}-\left(1-\frac{r^{2} r_{g}}{a^{3}}\right)^{1 / 2}\right] \ddot{t}+\dot{r} \dot{t} \frac{r r_{g}}{a^{3}}\left(1-\frac{r^{2} r_{g}}{a^{3}}\right)^{-1 / 2}=0
$$

Equation (9) is the time equation of motion for test particles in this gravitational field.

Similarly, setting $\mu=1$ in Equation (3) gives:

$$
\frac{d^{2} x^{1}}{d \tau^{2}}+\Gamma_{33}^{1}\left(\frac{d x^{3}}{d \tau}\right)\left(\frac{d x^{3}}{d \tau}\right)+\Gamma_{11}^{1}\left(\frac{d x^{1}}{d \tau}\right)\left(\frac{d x^{1}}{d \tau}\right)+\Gamma_{22}^{1}\left(\frac{d x^{2}}{d \tau}\right)\left(\frac{d x^{2}}{d \tau}\right)=0
$$

and substituting the explicit expressions of the affine connection coefficients gives

$$
\ddot{r}+r \sin ^{2} \theta\left(\frac{a^{3}-r^{2} r_{g}}{a^{3}}\right) \dot{\phi}^{2}+\frac{r r_{g}}{a^{3}}\left(\frac{a^{3}}{a^{3}-r^{2} r_{g}}\right) \dot{r}^{2}-r\left(\frac{a^{3}-r^{2} r_{g}}{a^{3}}\right) \dot{\theta}^{2}=0
$$

This is the radial equation of motion of stellar particles

Also for $\mu=2$ and $\mu=3$ in Equation (3), the polar and azimuthal equations of motion in the inner gravitational field of a spherical liquid star are obtained respectively as:

$$
\ddot{\theta}+\frac{\dot{r} \dot{\theta}}{r}-\sin \theta \cos \theta \cdot \dot{\phi}^{2}=0
$$

and

$$
\ddot{\phi}+\cot \theta \cdot \dot{\theta} \dot{\phi}+\frac{\dot{r} \dot{\phi}}{r}=0
$$

The radial geodesic Equation (11) is explicitly space related unlike Schwarzschild radial equation of motion which is space-time related ${ }^{12}$.

Note that when $\mu=0$, the geodesic equation of motion (9) is explicitly a function of radial and time coor- 
dinate derivatives of proper time. Geodesic equation with $\mu=1,2,3$ are purely space related.

Now, consider stellar particles moving in the equatorial plane $(\theta=\pi / 2)$ of the spherical liquid star. Thus, Equation (1) reduces to:

$c^{2}=\frac{1}{4} c^{2}\left[3\left(1-\frac{r_{g}}{a}\right)^{1 / 2}-\left(1-\frac{r^{2} r_{g}}{a^{3}}\right)^{1 / 2}\right]^{2} \dot{t}^{2}-\left(1-\frac{r^{2} r_{g}}{a^{3}}\right)^{-1} \dot{r}^{2}-r^{2} \dot{\phi}^{2}$

Explicit expressions for $\dot{t}$ and $\dot{\phi}$ are obtained respectively by solving the geodesic Equations (9) and (13).

From the time - like geodesic Equation (9) we can write:

$$
\frac{\ddot{t}}{\dot{t}}+\frac{\frac{\dot{r} r r_{g}}{a^{3}}\left(1-\frac{r^{2} r_{g}}{a^{3}}\right)^{-1 / 2}}{3\left(1-\frac{r_{g}}{a^{3}}\right)^{1 / 2}-\left(1-\frac{r^{2} r_{g}}{a^{3}}\right)^{1 / 2}}=0
$$

and integrating (15) yields:

$$
\dot{t}=\frac{A}{3\left(1-\frac{r_{g}}{a}\right)^{1 / 2}-\left(1-\frac{r^{2} r_{g}}{a^{3}}\right)^{1 / 2}}
$$

Where $A$ is a constant.

Considering the geodesic Equation (13) at the equator, it can be shown that:

$$
\dot{\phi}=\frac{l}{r}
$$

Now, writing Equation (14) in terms of (16) and (17) gives:

$c^{2}=\frac{1}{4} c^{2} A\left[3\left(1-\frac{r_{g}}{a}\right)^{1 / 2}-\left(1-\frac{r^{2} r_{g}}{a^{3}}\right)^{1 / 2}\right]-\left(1-\frac{r^{2} r_{g}}{a^{3}}\right)^{-1} \dot{r}^{2}-l$
Where $l$ is a constant.

Equation (18) can be written in a simplified form as:

$$
\dot{r}=x\left[\frac{1}{4} A c^{2}(\alpha-x)-l+c^{2}\right]^{1 / 2}
$$

Where

$$
3\left(1-\frac{r_{g}}{a}\right)^{1 / 2}=\alpha
$$

And

$$
\left(1-\frac{r^{2} r_{g}}{a^{3}}\right)^{1 / 2}=x
$$

Differentiating Equation (19) with respect to proper time we obtain:

$\ddot{r}=\left[x\left(\frac{1}{4} A c^{2}(\alpha-x)-l-c^{2}\right)-\frac{1}{8} A c^{2} x^{2}\right] \frac{d x}{d r}$

It can be deduced from Equation (20b) that:

$$
\frac{d x}{d r}=-\frac{r r_{g}}{a^{3} x}
$$

And hence Equation (21) becomes:

$$
\ddot{r}=-\frac{r r_{g}}{a^{3} x}\left[x\left(\frac{1}{4} A c^{2}(\alpha-x)-l-c^{2}\right)-\frac{1}{8} A c^{2} x^{2}\right]
$$

Expressing $\ddot{r}$ on the left hand side of (23) as a function of $\phi$ using the substitution $r=1 / u(\theta)$, yields:

$$
\ddot{r}=\frac{l}{u}\left(\frac{d u}{d \phi}\right)^{2}-l \frac{d^{2} u}{d \phi^{2}}
$$


Equation (23) can thus be written as:

$\frac{d^{2} u}{d \phi^{2}}-\frac{1}{u}\left(\frac{d u}{d \phi}\right)^{2}=\frac{r r_{g}}{l a^{3} x}\left[x\left(\frac{1}{4} A c^{2}(\alpha-x)-l-c^{2}\right)-\frac{1}{8} A c^{2} x^{2}\right]$

Equation (25) is the equation of motion of stellar particles of non-zero rest masses along the equatorial plane of a spherical liquid star. The $2^{\text {nd }}$ and $4^{\text {th }}$ terms on the right hand side are contributions from general relativity and the liquid nature of the star, while the $1^{\text {st }}$ and $3^{\text {rd }}$ terms are regular terms obtained in Newtonian theory for a spherical mass.

\section{Motion of Photons within a Spherical Liquid Star}

According to the General Theory of Relativity, light (photon) moves along a curve that has zero interval (null geodesic), i.e.

$$
d s^{2}=c^{2} d \tau^{2}=0
$$

The implication of Equation (26) is that photon or light is timeless.

Now, considering a photon moving in the equatorial plane, Equations (1) and (26) yield:

$0=\frac{1}{4}\left(3 \sqrt{1-\frac{r_{g}}{a}}-\sqrt{1-\frac{r^{2} r_{g}}{a^{3}}}\right)^{2} c^{2} d t^{2}-\frac{d r^{2}}{1-\frac{r^{2} r_{g}}{a^{3}}}-r^{2} d \phi^{2}$

Describing the motion of the photon with respect to a parameter $\omega$, and dividing Equation (27) by $d \omega^{2}$ all through yields;

$0=\frac{c^{2}}{4}\left(3 \sqrt{1-\frac{r_{g}}{a}}-\sqrt{1-\frac{r^{2} r_{g}}{a^{3}}}\right)^{2}\left(\frac{d t}{d \omega}\right)^{2}-\left(1-\frac{r^{2} r_{g}}{a^{3}}\right)^{-1}\left(\frac{d r}{d \omega}\right)^{2}-r^{2}\left(\frac{d \phi}{d \omega}\right)^{2}$
Or

$$
0=\frac{c^{2}}{4}\left(3 \sqrt{1-\frac{r_{g}}{a}}-\sqrt{1-\frac{r^{2} r_{g}}{a^{3}}}\right)^{2} \dot{t}^{2}-\left(1-\frac{r^{2} r_{g}}{a^{3}}\right)^{-1} \dot{r}^{2}-r^{2} \dot{\phi}^{2}
$$

Where $\dot{t}=d t / d \omega, \dot{r}=d r / d \omega$ and $\dot{\phi}=d \phi / d \omega$.

Substituting Equations (16) and (17) into (29) yields:

$$
0=\frac{A c^{2}}{4}-\left(1-\frac{r^{2} r_{g}}{a^{3}}\right)^{-1} \dot{r}^{2}-l
$$

Expressing $r$ as a function of $\phi$ i.e $r=r(\phi)$, then $\dot{r}=\dot{\phi} d r / d \phi$ and Equation (30) will become:

$\dot{\phi}^{2}\left(\frac{d r}{d \phi}\right)^{2}=\frac{A c^{2}}{4}\left(1-\frac{r^{2} r_{g}}{a^{3}}\right)-\left(1-\frac{r^{2} r_{g}}{a^{3}}\right) l$

Letting $r(\phi)=1 / u(\phi)$ and using equation (17), it can be shown that equation (31) takes the form:

$$
\frac{d u}{d \phi}=u\left(1-\frac{r_{g}}{u^{2} a^{3}}\right)^{1 / 2}\left(\frac{A c^{2}}{4 l}-1\right)^{1 / 2}
$$

Differentiating Equation (32) with respect to $\phi$ yields:

$$
\frac{d^{2} u}{d \phi^{2}}=\left(\frac{A c^{2}}{4 l}-1\right)^{1 / 2}\left[\left(1-\frac{r_{g}}{u^{2} a^{3}}\right)^{1 / 2}-\frac{r_{g}}{u^{2} a^{3}}\left(1-\frac{r_{g}}{u^{2} a^{3}}\right)^{-1 / 2}\right] \frac{d u}{d \phi}
$$

Equation (33) is the relativistic equation of motion of a photon in the equatorial plane of a spherical liquid star.

The motion of a photon in the equatorial plane of a spherical liquid star is similar to equation of motion of a 
photon obtained from Schwarzschild metric of a masspoint; though the Hilbert radius differs in the two cases.

\section{Conclusion}

The inner space metric of a spherical liquid star is exploited to deduce the equation of motion of stellar particles within a star. Also, the equation of motion of a photon is obtained from the space metric by using the condition that light (photon) travels along a null geodesic.

These equations can be solved to appreciate the behavior of stellar particles and photons as they move within spherical liquid stars as compared to the external field which is well known to be that of Schwarz child's masspoint metric. This work can also be extended to study the mechanics of spheroidal stars that abound in the universe.

\section{References}

1. Eddington AS. The internal constitution of the stars. 1st ed. Cambridge: Cambridge University Press; 1926.

2. Robitaille PM. A high temperature liquid plasma model of the Sun. Progress in Physics. 2007; 3(1):70-81.

3. Borissova L. The gravitational field of a condensed matter model of the Sun: The space breaking meets the Asteroid strip. Abraham Zelmanov Journal. 2009; 2:224-60.
4. Robitaille PM. Forty lines of evidence for condensed matter: Liquid metallic hydrogen as a building block for the Sun. Progress in Physics. 2013; 9(4):67-9.

5. Borrisova L, Rabounski D. Inside Stars. Rehoboth New Mexico; 2014.

6. Schwarzschild K. On the gravitational field of a point mass according to Einstein's theory. Abraham Zelmanov Journal. 2008; 1:10-9.

7. Schwarzschild K. On the gravitational field of a sphere of incompressible liquid, according to Einstein's theory. Abraham Zelmanov Journal. 2008; 1:20-32.

8. Sitter WD. On the curvature of space. Koninklijke Nederlandsche Akademie van Wetenschappen Proceedings; 1918. p. 229-43.

9. Ndikilar CE. Orbits in homogeneous time varying spherical spacetime. Progress in Physics. 2014; 10:52-5.

10. Ndikilar CE. Gravitational fields of conical mass distributions. Journal of Gravity. 2013; 2013:1-4.

11. Ndikilar CE, Howusu SXK. Gravitational radiation and propagation field equation exterior to astrophysical real or hypothetical time varying distributions of mass within regions of spherical geometry. Physics Essays. 2009; 22(1):73-7. https://doi.org/10.4006/1.3075827

12. Ndikilar CE. Gravitational fields exterior to homogeneous spheroidal masses. Abraham Zelmanov Journal. 2012; 5:31-67. 